\title{
Neurotrophic factor and Trk signaling mechanisms underlying the promotion of motor recovery after acute spinal cord injury in rats
}

\author{
HUA FANG $^{1 *}$, CHONG LIU $^{1 *}$, MIAO YANG ${ }^{1}$, HUAFENG LI ${ }^{2}$, FANGXIANG ZHANG ${ }^{1}$, \\ WEIJING ZHANG ${ }^{1}$ and JIANPING ZHANG ${ }^{1}$ \\ ${ }^{1}$ Department of Anesthesiology, Guizhou Provincial People's Hospital, Guiyang, Guizhou 550002; \\ ${ }^{2}$ Department of Anesthesiology, West China Second University Hospital of Sichuan University, \\ Chengdu, Sichuan 610041, P.R. China
}

Received September 14, 2016; Accepted March 8, 2017

DOI: $10.3892 / \mathrm{etm} .2017 .4516$

\begin{abstract}
Neurotrophic factor (NF) and Trk signaling mechanisms underlying the promotion of motor recovery following acute spinal cord injury (SCI) in rats were investigated. Thirty-six adult Sprague-Dawley rats of both genders were randomly divided into three groups: Sham-operated, model, and NF/Trk. Each group consisted of 12 rats, with four subgroups in each group: 1,3,5 and 7 days. Sham-operated rats received a laminectomy without SCI, while in model group rats, SCI was induced using an improved version of the Allen's method. After analepsia, sham-operated and model group rats were given normal saline via gavage, while the NF/Trk group received NFs and Trk. Lower limb function was measured using the Basso, Beattie and Bresnahan scale 1, 3,5 and 7 days before and after surgery. Results were analyzed statistically. Six rats from each group were randomly selected for sacrifice at 1,3,5 and 7 days after the operation. Morphological changes in motor neurons in the anterior gray column were observed by hematoxylin and eosin, and Nissl staining. Brain-derived expression of NF (BNDF) and neurotrophin-3 (NT-3) was detected by immunofluorescence, and the number of positive cells was counted. Expression of Trk B and Trk protein $\mathrm{C}$ receptor was measured by western blotting. In the NF/Trk group, the expression of NF/Trk pathway components remarkably increased. In addition, the morphology of motor neurons in the anterior gray column was improved. Expression of BNDF and NT-3 was significantly increased in motor neurons of the anterior gray column in NF/Trk rats compared with
\end{abstract}

Correspondence to: Dr Jianping Zhang, Department of Anesthesiology, Guizhou Provincial People's Hospital, 83 Zhongshan East Road, Guiyang, Guizhou 550002, P.R. China

E-mail: succiniter@126.com; oy8866086@163.com

${ }^{*}$ Contributed equally

Key words: spinal cord injury, neurotrophic factors, Trk signaling, motor neurons, anterior gray column those of sham-operated and model rats $(\mathrm{P}<0.05)$. NFs promote motor recovery following acute SCI in rats and may have valuable clinical applications.

\section{Introduction}

Spinal cord injury (SCI) includes primary and secondary SCI (1). Presently, there is no ideal treatment for SCI, creating a heavy burden for patients, their families, and the society $(2,3)$. The pathological and physiological responses to SCI are very complex. They include a sequence of cascade reactions, such as spinal cord edema, ischemia, ion imbalance, electrolyte disturbances, excitatory amino acid toxicity, inflammation, cell necrosis, and apoptosis (4).

Neurotrophic factors (NFs) are a family of polypeptide factors that support cell survival, promote cell growth and differentiation, and maintain normal cell function. NFs are important for spinal cord recovery, neuronal survival and regeneration, synaptic regeneration, and limb motor function after SCI (5-8). However, the effect of NFs after SCI has not been well defined. The mechanisms underlying NF-mediated neuronal recovery after SCI need to be explored further.

\section{Materials and methods}

Materials. Taq Master Mix (SinoBio USA, Walpole, MA, USA), agarose (Biowest, Nuaillé, France), sterile double-distilled water, anti-phospho-Trk receptor B (1:1,000; Cell Signaling Technology, Inc., Beverly, MA, USA), Trk receptor B antibody (1:5,000; Invitrogen, Carlsbad, CA, USA), 0.9\% sterile normal saline (Otsuka Pharmaceutical Co., Ltd., Tokyo, Japan), and NFs.

Instruments. PCR amplification and transilluminator (both from Bio-Rad, Berkeley, CA, USA), electrophoresis apparatus (Beijing Liuyi Biotechnology Co., Ltd., Beijing, China), centrifuge and micro-pipette (both from Eppendorf AG, Hamburg), Haier ice maker, western blotting electrophoresis apparatus (Bio-Rad), $-80^{\circ} \mathrm{C}$ freezer (Thermo Fisher Scientific, Waltham, MA, USA), $10 \mathrm{ml}$ syringe, $5 \mathrm{ml}$ syringe (Tianjin Hanaco Medical Co., Ltd., Tianjin, China), surgical 


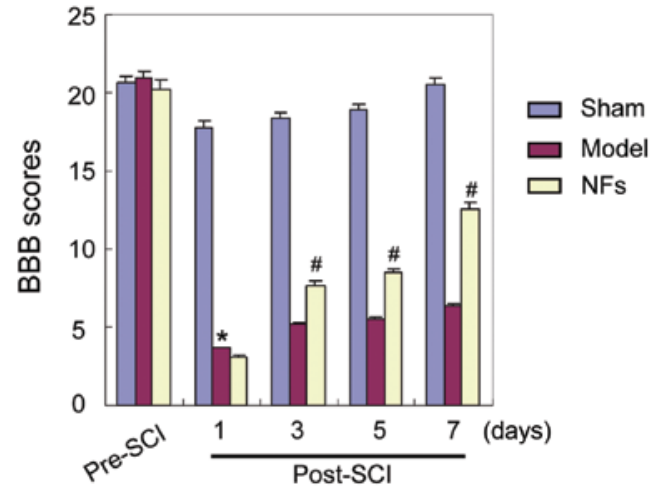

Figure 1. BBB scores for three different groups before and after SCI modeling. BBB scale scores of SCI rats in the model and NF/Trk groups clearly decreased, indicating that SCI was successfully induced in this model $\left({ }^{*} \mathrm{P}<0.05\right)$. BBB scale scores were not significantly different between the model and NF/Trk groups 1 day after the operation, but the BBB scale scores were significantly higher in NFs/Trk rats than in model rats 3,5 and 7 days after the operation $\left({ }^{\#} \mathrm{P}<0.05\right)$. BBB, Basso, Beattie and Bresnahan scale; $\mathrm{SCI}$, spinal cord injury; NF, neurotrophic factor.

instruments for use on laboratory animals (Beijing Medical Device Factory, Beijing, China), NanoDrop2000 photometric analyzer (Thermo Fisher Scientific, Wilmington, DE, USA), Eppendorf tube (Eppendorf AG), water bath (Beijing Medical Device Factory), and pathology microtome (Leica, Mannheim, Germany).

\section{Research methods}

Animals and surgical procedure. Laboratory animal grouping: Seventy-two adult Sprague-Dawley rats were randomly assigned to three groups regardless of gender: Sham-operated, model, and NFs/Trk groups. There were 24 rats in each group. For each group, four subgroups (1,3,5 and 7 days) were created with respect to the survival time. Sham-operated rats were operated with a T10 laminectomy without SCI. The rats were given normal saline via gavage after analepsia. Motor function was measured using the Basso, Beattie and Bresnahan (BBB) scale $1,3,5$ and 7 days after the operation in all groups. Rats from all groups were sacrificed for experiments after motor function was assessed. Rats in the model group received a laminectomy and SCI was induced using Allen's method (9). In the NF/Trk group, rats were operated in the same way as the model group, but received NF/Trk by gavage with normal saline after analepsia. This study was approved by the Animal Ethics Committee of Guizhou Provincial People's Hospital.

Evaluation of neuronal morphology and NT expression. Changes in neuronal morphology were observed by hematoxylin and eosin staining and Nissl staining. Expression of brain-derived NF (BNDF) and neurotrophin-3 (NT-3) was detected by immunofluorescence staining and the number of positive cells was calculated based on semi-quantitative analysis. Expression of Trk receptor B and C was tested by western blotting.

BBB locomotor scale. The BBB locomotor scale is a scoring system that tests the motor function of rats with SCI. Scores range from 1 to 21 , and the higher the score, the better the motor function of the lower limb.
A

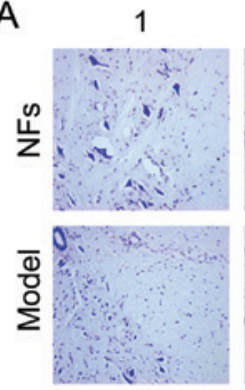
3

5

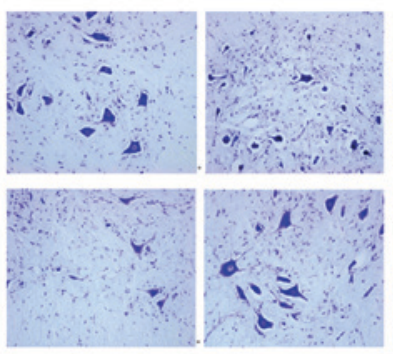

B

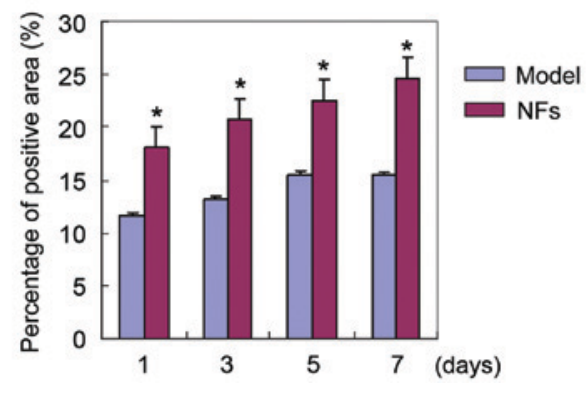

Figure 2. Nissl staining for model and NF groups. (A) Nissl staining results of rat spinal cord neurons observed by a x50 inverted microscope. (B) Neuronal injury in the NF/Trk group was significantly less severe than that in the model group $(\mathrm{P}<0.05)$. NF, neurotrophic factor.

Statistical analysis. Statistical analysis was performed using SPSS 19.0 (IBM, Armonk, NY, USA). Qualitative data were analyzed by analysis of variance (ANOVA) and $\chi^{2}$ test, and data that did not meet the conditions for a $2 \times 2$ table were analyzed by Fisher's exact test. Quantitative data were compared by ANOVA, and a P-value of $<0.05$ was considered to indicate a statistically significant difference.

\section{Results}

BBB locomotor scale. The BBB locomotor scale was used to assess the limb motor function of rats with SCI. The results indicated no significant difference in limb motor function between the three groups before treatment. In the sham-operated group, there was no significant difference in motor function before and after the operation. In the groups of model and NFs/Trk rats, the BBB scale scores decreased significantly after the operation $(\mathrm{P}<0.05)$. However, as shown in Fig. 1, the BBB scale scores were significantly higher in the NF/Trk group compared with those in the model group at all tested time-points. In addition, rats in the NFs/Trk group recovered significantly faster after the operation (Fig. 1) $(\mathrm{P}<0.05)$.

Nissl staining of spinal cord tissue. Nissl staining was used to examine the injury of spinal cord tissue in SCI rats. Nissl bodies were stained with purple-blue, which identified the basic structure of spinal cord neurons. Large and numerous Nissl bodies indicate strong protein synthesis in neural cells, while Nissl bodies are reduced or absent in injured neurons. Nissl staining revealed that varying degrees of neuronal injury in the spinal cord of NF/Trk and model rats after SCI was induced. However, neuronal injury in the NFs/Trk group was significantly less severe than that in the model group (Fig. 2) $(\mathrm{P}<0.05)$. 
A
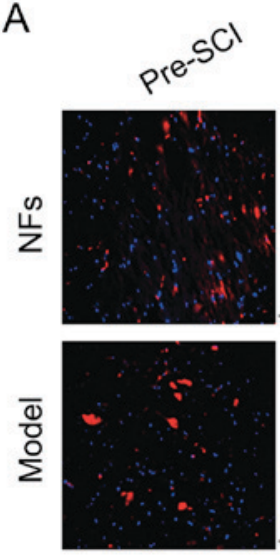

B
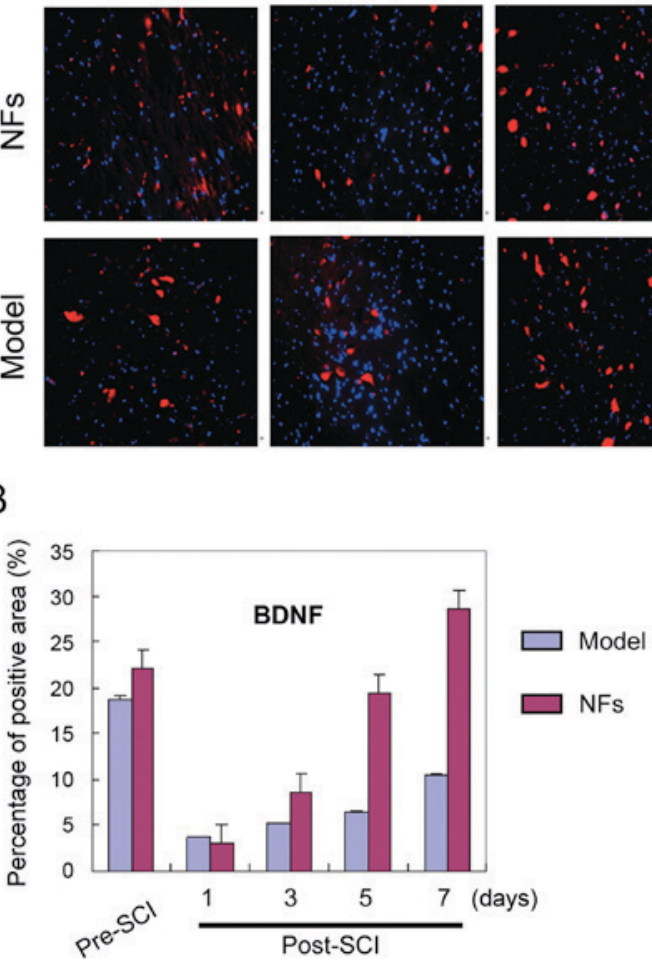

Post-SCI

\begin{tabular}{llll}
\hline 1 & 3 & 5 & \\
\hline
\end{tabular}
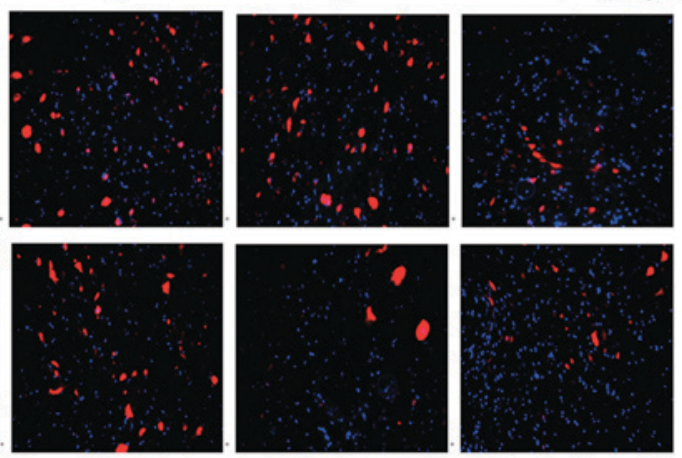

$\mathrm{C}$

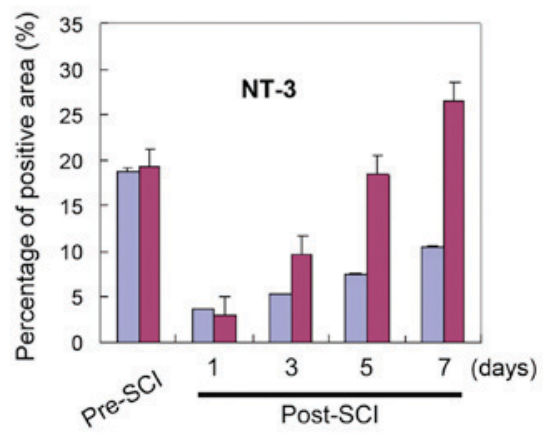

Figure 3. BNDF and NT-3 expression in spinal cord neurons. (A) Immunofluorescence staining results. BNDF (blue) and NT-3 (red)-positive cells in the spinal cord tissue of model and NF/Trk group rats. (B and C) BDNF- and NT-3-positive cells were quantified in rat spinal cord tissues and significantly more positive neurons were detected in NF/Trk rats than in model group rats $(\mathrm{P}<0.05)$. BNDF, brain-derived neurotrophic factor; NT-3, neurotrophin-3; NF, neurotrophic factor.

A

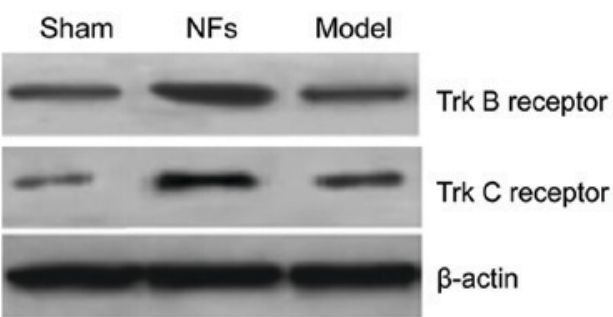

B

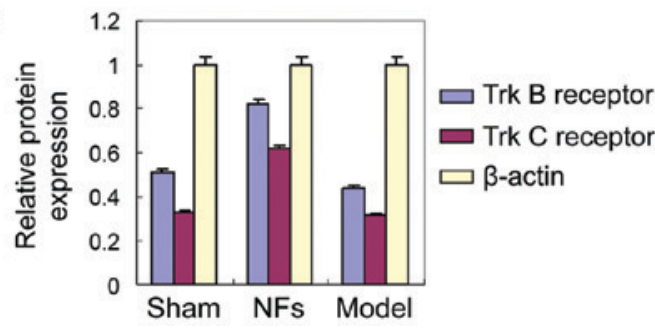

Figure 4. Expression of Trk B and Trk C receptors. (A) Western blot assay was performed to detect expression of Trk B and Trk C receptors. (B) Trk B and Trk $\mathrm{C}$ receptors in NF group were significantly higher than model group. NFs, neurotrophic factors.

BNDF and NT-3 expression in rat spinal cord tissue. BNDF and NT-3 staining were visualized in the spinal cord tissue of model and NFs/Trk rats. Immunofluorescence experiments showed significantly more BDNF and NT-3-positive cells in the spinal cord of NFs/Trk rats compared to those in model rats (Fig. 3) $(\mathrm{P}<0.05)$. BDNF and NT-3 were mainly localized in neurons of spinal cord tissue.
Trk $B$ and Trk $C$ receptor expression in rat spinal cord tissue. To investigate the role of NF signaling in SCI recovery, the expressions of Trk B and Trk $\mathrm{C}$ receptors were analyzed by western blotting in the injured rat spinal cord with and without NF/Trk treatment. The expressions of Trk B and Trk C receptors were significantly higher in the spinal cord of NFs/Trk rats than model group rats (Fig. 4) $(\mathrm{P}<0.05)$. This finding indicates that NFs promote recovery following SCI by activating the expressions of Trk B and Trk $\mathrm{C}$ receptors in injured rat spinal cord tissue.

\section{Discussion}

The spinal cord can be directly or indirectly injured by external force, causing motor, sense, and sphincter dysfunction, abnormal muscle tone, and pathological changes (10-14). The degree and clinical characteristics of SCI depend on the primary source of the injury. The high incidence of traumatic SCI in young adults often results in limb motor dysfunction and multiple associated injuries. This can make treatment difficult and lead to many complications and disability. SCI represents a serious physical and psychological injury that creates an enormous burden for patients, their families, and society. Therefore, preclinical research of trauma surgery is both important and challenging (15-17).

Primary and secondary SCI induces a sequence of cellular and molecular pathological events. Secondary injury includes an inflammatory response, which increases the severity of SCI. Both primary and secondary SCI can cause paralysis 
or death. SCI can trigger pathological and physical events. Correlations between the primary mechanical injury and the secondary injury promote pathological changes $(4,5)$. SCI is often followed by local and systemic inflammation, which increases the pathogenesis of the injury (6). In addition, the expression of certain genes is increased, which may mediate the inflammatory response. These genes belong to signaling pathways including $\mathrm{NF}-\kappa \mathrm{B}$ and adenosine monophosphateactivated protein kinase.

Nerve growth factor (NGF) is a regulator of nerve growth and mediates neurological nutrition and neurite outgrowth. NGF is often released after SCI. NGF was the first NF to be described and has been thoroughly studied. It has important regulatory effects on the development, differentiation, growth, regeneration, and function of central and peripheral neurons. The NF family is very large and includes NGF, BDNF, basic fibroblast growth factor (bFGF), ciliary NF, and NT-3 (18-21).

NFs can promote recovery after SCI by multiple mechanisms. The main effects and mechanisms are: i) Inhibition of c-fos and c-jun expressions in the spinal cord, thereby reducing apoptosis of spinal cord nerve cells; ii) decreasing TNF- $\alpha$, IL- $1 \beta$, and IL- 8 expressions in the spinal cord, inhibiting the inflammatory response; and iii) reduction of inflammation and spinal cord ischemia-reperfusion injury by inhibiting $\mathrm{NF}-\kappa \mathrm{B}$ and VCAM-1.

In this study, we induced SCI in rats using the Allen's method, and investigated the effect of NF treatment on SCI recovery. We demonstrated that limb motor function was significantly improved after SCI by NF treatment 3 days after injury. This suggests that NFs can effectively improve motor neuron function, preserve uninjured spinal cord tissues, and accelerate functional recovery in rats following SCI. Nissl staining of injured spinal cord tissue indicated that neuronal injuries were less severe following NF treatment compared with those after saline treatment. These findings demonstrated that NFs could effectively protect neuronal tissues after injury.

To explore the mechanism of NF action after SCI, we examined expression of BNDF and NT-3 in spinal cord tissue after NF and saline treatment. NF treatment significantly increased the number of BDNF and NT-3-positive cells in rat spinal cord tissue after injury. This finding is consistent with previous published results. For example, Zhang et al treated rabbits with NFs after ischemia-reperfusion injury and observed satisfactory recovery (22).

BDNF and NT-3 were predominantly expressed in spinal cord neurons. BDNF and NT-3 are members of the NGF family and are weakly expressed under normal conditions. Following neuronal injury, multiple inflammatory factors are stimulated and the expression of BDNF, NT-3, and other NGFs increases, initiating neuronal protective mechanisms through multiple upstream and downstream signaling pathways. This protects uninjured neurons and promotes the growth and repair of injured nerve tissue. Clinical findings have shown that NGF concentrations correlate positively with the recovery of nerve functions. This is because the extent of self-recovery of nerve tissues was limited. Increasing NGF levels can promote selfrecovery and improve prognosis, which is consistent with the present findings.

We demonstrated that the neuroprotective effect of NFs was caused by enhanced expression of Trk B and Trk C receptors in the injured rat spinal cord. The Trk receptor family is a family of receptor tyrosine kinase that regulates the strength and plasticity of synapses in the mammalian nervous system (1). The activation of Trk receptors affects the survival, differentiation, and function of neurons through multiple signaling pathways. NF is a common ligand of Trk receptors and plays a critical role in the nervous system $(2,23)$. The binding between NFs and Trk receptors is highly specific. Each NF has a corresponding Trk receptor, with a different affinity. The signaling pathway that is induced when NF binds to a Trk receptor regulates cell survival and function. Therefore, NFs improve recovery of rat spinal cord tissue after injury by promoting Trk receptor expression, thereby increasing NGF binding to Trk receptors and enhancing the effect of NGF.

In conclusion, we have demonstrated that NFs can promote recovery of motor function after acute SCI in rats through $\mathrm{NF}$ /Trk pathways. NFs support the survival, regeneration, and axonal repair of neurons and improve motor function after SCI by promoting BDNF, NGF, and NT-3 expression and activation of their high-affinity receptors.

\section{Acknowledgements}

This study was supported by the National Key Technology R\&D Program (2014BAI05B05), the Foundation of Science and Technology Department of Guizhou Province (Qiankehe SY zi[2012]3090), the Foundation of Science and Technology Department of Guizhou Province (Qiankehe SY zi[2013]3063), and the Foundation of Science and Technology Department of Guizhou Province (Qiankehe LH zi[2014]7021).

\section{References}

1. Tashiro S, Nishimura S, Iwai H, Sugai K, Zhang L, Shinozaki M, Iwanami A, Toyama Y, Liu M,Okano H, et al: Functional recovery from neural stem/progenitor cell transplantation combined with treadmill training in mice with chronic spinal cord injury. Sci Rep 6: 30898, 2016.

2. Baaj AA, Uribe JS, Nichols TA, Theodore N, Crawford NR, Sonntag VK and Vale FL: Health care burden of cervical spine fractures in the United States: analysis of a nationwide database over a 10-year period. J Neurosurg Spine 13: 61-66, 2010.

3. Pickelsimer E, Shiroma EJ and Wilson DA: Statewide investigation of medically attended adverse health conditions of persons with spinal cord injury. J Spinal Cord Med 33: 221-231, 2010.

4. Volarevic V, Erceg S, Bhattacharya SS, Stojkovic P, Horner P and Stojkovic M: Stem cell-based therapy for spinal cord injury. Cell Transplant 22: 1309-1323, 2013.

5. Kazim SF and Iqbal K: Neurotrophic factor small-molecule mimetics mediated neuroregeneration and synaptic repair: emerging therapeutic modality for Alzheimer's disease. Mol Neurodegener 11: 50, 2016.

6. Fernandes BS, Molendijk ML, Köhler CA, Soares JC, Leite CM, Machado-Vieira R, Ribeiro TL, Silva JC, Sales PM, Quevedo J, et al: Peripheral brain-derived neurotrophic factor (BDNF) as a biomarker in bipolar disorder: a meta-analysis of 52 studies. BMC Med 13: 289, 2015.

7. Cook DJ, Nguyen C, Chun HN, L Llorente I, Chiu AS, Machnicki M, Zarembinski TI and Carmichael ST: Hydrogel-delivered brain-derived neurotrophic factor promotes tissue repair and recovery after stroke. J Cereb Blood Flow Metab: May 12, 2016 (Epub ahead of print).

8. Zhong Z, Gu H, Peng J, Wang W, Johnstone BH, March KL, Farlow MR and Du Y: GDNF secreted from adipose-derived stem cells stimulates VEGF-independent angiogenesis. Oncotarget 7: 36829-36841, 2016.

9. Koozekanani SH, Vise WM, Hashemi RM and McGhee RB: Possible mechanisms for observed pathophysiological variability in experimental spinal cord injury by the method of Allen. J Neurosurg 44: 429-434, 1976. 
10. Yang L, Ge Y, Tang J, Yuan J, Ge D, Chen H, Zhang H and Cao X: Schwann cells transplantation improves locomotor recovery in rat models with spinal cord injury: a systematic review and meta-analysis. Cell Physiol Biochem 37: 2171-2182, 2015.

11. Yüksekkaya M, Tutar N, Büyükoğlan H, Dündar M, Yılmaz İ, Gülmez I, Oymak FS, Balta B, Korkmaz K and Demir R: The association of brain-derived neurotrophic factor gene polymorphism with obstructive sleep apnea syndrome and obesity. Lung 194: 839-846, 2016.

12. Wang X, Peng B, Xu C, Gao Z, Cao Y, Liu Z and Liu T: BDNF-ERK1/2 signaling pathway in ketamine-associated lower urinary tract symptoms. Int Urol Nephrol 48: 1387-1393, 2016.

13. Adhikary S, Li H, Heller J, Skarica M, Zhang M, Ganea D and Tuma RF: Modulation of inflammatory responses by a cannabinoid-2-selective agonist after spinal cord injury. J Neurotrauma 28: 2417-2427, 2011.

14. Chiu WT, Lin HC, Lam C, Chu SF, Chiang YH and Tsai SH: Review paper: epidemiology of traumatic spinal cord injury: comparisons between developed and developing countries. Asia Pac J Public Health 22: 9-18, 2010.

15. Cuzzocrea S, Riley DP, Caputi AP and Salvemini D: Antioxidant therapy: a new pharmacological approach in shock, inflammation, and ischemia/reperfusion injury. Pharmacol Rev 53: 135-159, 2001.

16. Kernie SG and Parent JM: Forebrain neurogenesis after focal ischemic and traumatic brain injury. Neurobiol Dis 37: $267-274,2010$.

17. Freund P, Weiskopf N, Ward NS, Hutton C, Gall A, Ciccarelli O, Craggs M, Friston K and Thompson AJ: Disability, atrophy and cortical reorganization following spinal cord injury. Brain 134: $1610-1622,2011$.
18. Boyce VS, Tumolo M, Fischer I, Murray M and Lemay MA Neurotrophic factors promote and enhance locomotor recovery in untrained spinalized cats. J Neurophysiol 98: 1988-1996, 2007.

19. Garraway SM, Woller SA, Huie JR, Hartman JJ, Hook MA, Miranda RC, Huang YJ, Ferguson AR and Grau JW: Peripheral noxious stimulation reduces withdrawal threshold to mechanical stimuli after spinal cord injury: role of tumor necrosis factor alpha and apoptosis. Pain 155: 2344-2359, 2014.

20. Huie JR, Stuck ED, Lee KH, Irvine KA, Beattie MS, Bresnahan JC, Grau JW and Ferguson AR: AMPA receptor phosphorylation and synaptic colocalization on motor neurons drive maladaptive plasticity below complete spinal cord injury. eNeuro 2: pii: ENEURO.0091-15.2015, 2015.

21. Liu NK and Xu XM: Neuroprotection and its molecular mechanism following spinal cord injury. Neural Regen Res 7: 2051-2062, 2012

22. Zhang S, Tobaru T, Zivin JA and Shackelford DA: Activation of nuclear factor-kappaB in the rabbit spinal cord following ischemia and reperfusion. Brain Res Mol Brain Res 63: 121-132, 1998.

23. Paterniti I, Esposito E, Mazzon E, Bramanti P and Cuzzocrea S: Evidence for the role of PI(3)-kinase-AKT-eNOS signalling pathway in secondary inflammatory process after spinal cord compression injury in mice. Eur J Neurosci 33: 1411-1420, 2011. 